\title{
CASO -2018: Uso de bomba de baclofeno intratecal para el manejo de la espasticidad en lesión medular traumática
}

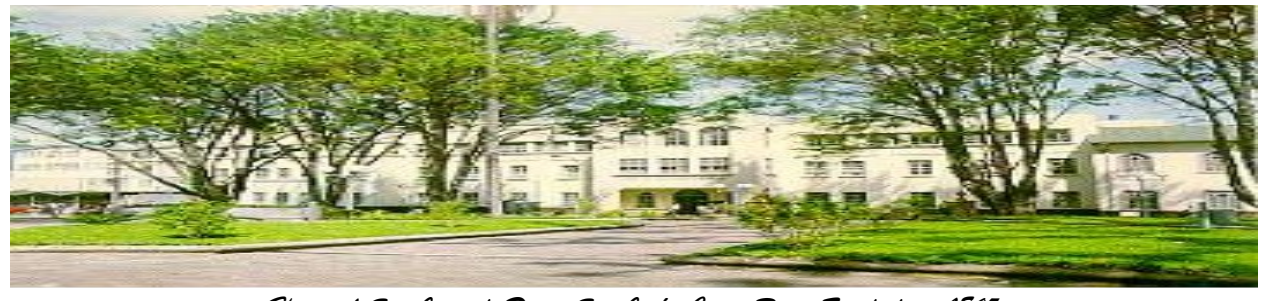

Haspital San Guan de Dios. San Dosé. Costa Rica. Fundado en 1845
ISSN

2215-

2741

Recibido:

$22 / 01 / 2018$

Aceptado:

$15 / 03 / 2018$

María Gabriela Flores López ${ }^{1}$

Griska Ledezma Cubero ${ }^{2}$

Alejandra Delgado ${ }^{3}$

\begin{abstract}
${ }^{1}$ Residente de Medicina Física y Rehabilitación, Centro Nacional de Rehabilitación. gafl3084@gmail.com. ${ }^{2}$ Asistente de Medicina Física y Rehabilitación, Centro Nacional de Rehabilitación. ledezmacubero@yahoo.com

${ }^{3}$ Asistente de Anestesiología y Algología Intervencionista, Centro Nacional de Rehabilitación. ale.delga$\underline{\text { magmail.com }}$
\end{abstract}

\section{RESUMEN}

La espasticidad secundaria a una lesión medular traumática, es un trastorno motor caracterizado por aumento del tono muscular velocidad dependiente, que genera secundariamente dolor crónico, úlceras por presión; entre otros. El tratamiento de esta patología se basa en programas de rehabilitación integral, terapia farmacológica y de ser necesario de intervenciones quirúrgicas. El baclofeno, es útil para el manejo de la espasticidad, inicialmente se usa vía oral, pero por su baja biodisponibilidad o mala tolerancia, se puede preferir la vía intratecal. El objetivo del presente artículo es presentar el reporte de un caso, de un paciente con diagnóstico de Tetraplejía Completa Bajo C3 ASIA A secundario a accidente de tránsito en el 2010, que recibió tratamiento con baclofeno intratecal, convirtiéndose en una opción segura $y$ eficaz para el manejo de la espasticidad severa y espasmos refractarios al manejo convencional.

\section{PALABRAS CLAVE}

Bomba de baclofeno. Espasticidad. Lesión medular. Baclofeno intratecal. Espasmos.
ABSTRACT
Spasticity secondary to a traumatic spinal cord injury is a motor disorder characterized by increased rate of muscle tone, which secondarily generates chronic pain, pressure ulcers; among others. The treatment of this pathology is based on programs of integral rehabilitation, pharmacological therapy and if necessary, 
surgical interventions. Baclofen, is useful for the management of spasticity, initially it's used orally, but because of its low bioavailability or poor tolerance, the intrathecal route may be preferred. The objective of the present article is to present the report of a case of a patient with diagnosis of complete tetraplegia under C3 ASIA A secondary to a traffic accident in 2010, who received treatment with intrathecal baclofen, becoming a safe and effective option for the management of severe spasticity and spasms refractory to conventional management.

\section{KEY WORDS}

Baclofen pump. Spasticity. Spinal cord injury. Intrathecal baclofen. Spasms.

\section{INTRODUCCIÓN}

La espasticidad fue definida por Lance ${ }^{(1)}$, como un trastorno motor desencadenado por una alteración en el sistema nervioso central (SNC), como parte del síndrome de neurona motora superior $^{(2)}$, que clínicamente se caracteriza por un aumento del tono muscular dependiente de la velocidad, con reflejos del estiramiento tónico (miotático) exagerados, presencia de espasmos, hiperreflexia osteotendinosa y aparición de posturas anormales ${ }^{(2,3,4)}$.

En personas que han sufrido una lesión medular, la presencia de espasticidad es una secuela frecuente; que al cronificarse es un importante problema de salud. Causando discapacidad significativa y deterioro en la calidad de vida; provocando dificultades para realizar transferencias, al auto cuidado e higiene; generando dolor, deformidad y alteraciones del sueño, e incluso puede llevar a incapacidades prolongadas ${ }^{(5,4)}$.

El objetivo del artículo es presentar la respuesta terapéutica de la implantación intratecal de baclofeno, en un paciente con espasmos y espasticidad secundaria a una lesión medular traumática de 6 años de evolución, con poca respuesta a la terapia oral.

\section{CASO CLÍNICO}

Presentamos el caso de un paciente masculino de 23 años, con Tetraplejía Completa Bajo C3 ASIA
A, secundaria a accidente de tránsito en bicicleta en el 2010, con fractura por estallido de C3 de manejo quirúrgico, quien posterior a cumplir internamiento de 22 días, para proceso de rehabilitación integral en el Centro Nacional de Rehabilitación (CENARE), en febrero del 2011, se egresa con Tono grado 2 en Miembros Inferiores, según la escala modificada de Ashworth y espasmos de Penn 2 (ver tabla 1), ameritando inicio de tratamiento con Tizanidina a $6 \mathrm{mg} \mathrm{c} / 6$ horas vía oral (V.O), continuando manejo en consulta externa de Lesionados Medulares en Fisiatría, cada 6 meses.

Tabla 1. Escala Modificada de Ashworth y clasificación de Espasmos de Penn

\begin{tabular}{|c|c|c|}
\hline Grado & $\begin{array}{l}\text { Respuesta al movimiento se- } \\
\text { gún Ashworth }\end{array}$ & $\begin{array}{l}\text { Descripción Es- } \\
\text { pasmo según Penn }\end{array}$ \\
\hline $\mathbf{0}$ & Tono muscular normal & $\begin{array}{l}\text { Ausencia de espas- } \\
\text { mos }\end{array}$ \\
\hline 1 & $\begin{array}{l}\text { Aumento ligero del tono, resis- } \\
\text { tencia mínima en los últimos } \\
\text { grados del rango de movimiento }\end{array}$ & $\begin{array}{l}\text { Precipitados por es- } \\
\text { tímulos (provoca- } \\
\text { dos) }\end{array}$ \\
\hline $1+$ & $\begin{array}{l}\text { Aumento ligero del tono muscu- } \\
\text { lar, con resistencia en menos de } \\
\text { la mitad del rango de movimien- } \\
\text { to }\end{array}$ & \\
\hline 2 & $\begin{array}{l}\text { Aumento del tono más marcado } \\
\text { en la mayor parte del rango de } \\
\text { movimiento, pero la extremidad } \\
\text { puede moverse fácilmente }\end{array}$ & $\begin{array}{l}\text { Espontáneos, me- } \\
\text { nos de un espasmo } \\
\text { por hora }\end{array}$ \\
\hline 3 & $\begin{array}{l}\text { Considerable aumento del tono } \\
\text { muscular, el movimiento pasivo } \\
\text { es difícil de realizar }\end{array}$ & $\begin{array}{l}\text { Espontáneos, uno o } \\
\text { más espasmos por } \\
\text { hora }\end{array}$ \\
\hline 4 & $\begin{array}{l}\text { Rigidez de la parte afectada en } \\
\text { flexión o extensión }\end{array}$ & $\begin{array}{l}\text { Espontáneos, más } \\
\text { de } 10 \text { espasmos por } \\
\text { hora }\end{array}$ \\
\hline
\end{tabular}

Fuente: Propia

Entre marzo 2011 y setiembre 2012 presenta Tono grado $2-3$, según escala modificada de Ashworth en Miembros Inferiores, que aumenta con la actividad (principalmente al sedestarse), inicia manejo con aplicación de toxina botulínica tipo A, en la clínica de Espasticidad a nivel de flexores de caderas (músculos psoas iliaco y recto anterior bilateral) para mejorar la postura. Durante el 2012 a 2013, al no obtener respuesta clínica esperada se modifica medicación, con aumento de Tizanidina hasta dosis máximas a 36 $\mathrm{mg}$ /día V.O y toxina botulínica a $1600 \mathrm{U}$ en el 2014. 
A pesar de la terapia indicada, requirió internamiento por aumento de los espasmos, a niveles máximos, Penn 4 (más 50 al día) y gran dificultad para el aseo perineal. En el mes de agosto del 2016 es valorado por el servicio de Anestesiología- Algología y se considera candidato para implantación de bomba intratecal con baclofeno, para manejo de la espasticidad.

El 29 noviembre del 2016, se decide realizar prueba de baclofeno intratecal a dosis de $50 \mathrm{mcg}$ a nivel espinal en el espacio L3-L4, donde se reporta por parte del Médico Fisiatra a las 2 y 4 horas: tono de miembros inferiores Flácido, no se desencadenan espasmos a la movilización, distancia intercondilar en flexión de $41 \mathrm{~cm}$, codos con extensión completa y rodillas con extensión completa bilateral, evidenciándose adecuada respuesta.

Se realiza procedimiento de implantación de bomba de baclofeno intratecal en diciembre del 2016, por parte de Anestesiología intervencionista y Neurocirugía, en el Hospital Max Peralta de Cartago. Dicho procedimiento se realizó según la técnica usual, en decúbito lateral y guiado por fluoroscopía, en un abordaje paramediano a nivel del espacio intratecal de L3-L4, en un ángulo de $30^{\circ}$. Se realiza un túnel para el catéter, dirigiéndose hacia donde se implantará la bomba debajo de la dermis; y se implanta la bomba Synchro-

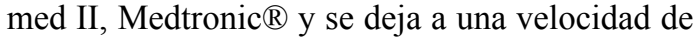
infusión $50 \mathrm{mcg}$ diarios.

En enero del 2017, el paciente reinicia con espasmos por lo que se aumenta la dosis de baclofeno a $75 \mathrm{mcg}$ y se suspende Tizanidina, un mes después se aumenta dosis al doble a $150 \mathrm{mcg}$. En marzo 2017 es valorado por Fisiatría con disminución de los espasmos a Penn 2 y Tono grado 2, según Ashworth modificado, el cual se ha mantenido hasta la fecha.

\section{DISCUSIÓN Y ANÁLISIS DEL CASO}

Este es el primer paciente manejado en el CENARE y de Costa Rica, con implantación de bomba de baclofeno para el tratamiento de la espasticidad refractaria, secundaria a una lesión medular traumática. Se logra evidenciar a esta terapia, como una buena alternativa al tratamiento usual con Tizanidina y Toxina botulínica, ya que el paciente no presentó mejoría en su evolución durante casi 6 años. A corto plazo luego de su colocación, se observa mejoría importante tanto en disminución del tono muscular y, principalmente, en la disminución de la frecuencia de los espasmos.

Ratificando lo expuesto en la literatura, donde se menciona que la infusión intratecal de baclofeno mediante bombas programables subcutáneas y catéter intratecales implantables; son una terapia alternativa a los antiespásticos orales y útiles para el manejo de la espasticidad generalizada, como un sistema seguro, bien tolerado y altamente eficaz. ${ }^{(6,7)}$ Considerando siempre el adecuar la dosis de la infusión de baclofeno, hasta alcanzar la dosis necesaria que logre la meta planteada en cada paciente.

En Estados Unidos se estiman una incidencia entre 10000 y 12000 casos nuevos de tetraplejía o paraplejía secundarios a lesión de la médula espinal (LME) ${ }^{(8)}$, siendo el 40 al 50\% lesiones traumáticas secundarias a accidentes de tránsito. Datos Europeos destacan el trabajo de Suecia, donde se menciona una incidencia de espasticidad secundaria a lesión medular traumática del 60$65 \%$ de pacientes, aumentando progresivamente luego del año ${ }^{(7)}$. Los patrones de localización de la espasticidad en la LME, luego del período de shock medular, van a ser dependientes del nivel y del grado lesional, según la clasificación internacional de las lesiones medulares, de la American Spinal Injury Association (ASIA), con una mayor incidencia y problemática de la espasticidad en pacientes incompletos cervicales, tipo ASIA B, C o D. Y van a mantener una tendencia hacia la cronicidad, por lo que se debe realizar un manejo lo más precoz posible ${ }^{(9)}$.

El baclofeno es un agonista del receptor GABA tipo B, que genera un control inhibidor pre- $\mathrm{y}$ postsináptico de la transmisión motora alterada por la lesión medular ${ }^{(7)}$, con inhibición a nivel espinal y supraespinal reduciendo la neurotransmisión excitatoria. ${ }^{(1,3)}$ Controla la espasticidad en el 70 al $87 \%$ de los pacientes y disminuye la frecuencia de espasmos ${ }^{(10)}$; con una vida media de $6 \mathrm{~h}$; por tanto su administración debe realizarse de manera continua. ${ }^{(5)}$

La infusión crónica de baclofeno dentro del espacio subaracnoideo, introducida en 1984 por 
Penn y Kroin ${ }^{(10)}$; permite alcanzar altas concentraciones del fármaco en el $\mathrm{SNC}$, sin mayores efectos adversos (debilidad muscular, somnolencia y alucinaciones). ${ }^{(2,3,9,4)}$

Los principales criterios de selección de pacientes para esta terapia son: espasticidad refractaria (poca reducción de la espasticidad con tratamiento usual, efectos secundarios no deseados, tono muscular grado 3 o $4^{(6,11)}$, valorados por un Fisiatra experimentado y según las escalas ya mencionadas para una valoración objetiva), cognitivamente íntegros y presencia de una buena red de apoyo; ya que de esto dependerá el éxito terapéutico.

\section{CONCLUSIONES}

El uso de bomba de baclofeno intratecal, en un paciente previamente bien seleccionado y con secuelas de una LME, evidenció ser un método seguro y bien tolerado a largo plazo, con mejoría significativa en el manejo del dolor asociado a la espasticidad; así como la frecuencia de los espasmos. Evita la progresión de las contracturas, presenta menor incidencia de efectos adversos; mejorando la calidad de vida y la funcionalidad de los pacientes.

\section{BIBLIOGRAFÍA}

1. Núñez S Poch J. Tratamiento de la espasticidad infantil mediante implante de bomba de infusión continua de baclofeno intratecal. Revista Latinoamericana de Neurocirugía. 2016; vol. $25 \mathrm{~N}^{\mathrm{o}} 1$.

2. Cáceres L Villa M Vera J. Bomba intratecal de baclofeno, ¿una intervención terapéutica útil y segura en pacientes con espasticidad? Presentación de casos. Revista IATREIA. Octubre-diciembre 2016; vol. 29(4): 478-484.

3. Chaná P Barrientos $\mathrm{N}$ Landerretche $\mathrm{J}$ et al. Manejo de la espasticidad en pacientes con lesión medular con infusión de baclofen intratecal mediante bomba implantable. Revista Chilena Neuro Psiquiatrica. 2001; 39(2): 149154.
4. Khurana S Garg D. Spasticity and the use of intratecal baclofen in patients with Spinal cord Injury. Physical Medicine Rehabilitation Clinics North America. 2014; vol. 25: 655-669.

5. Rivera R Arcila M Campuzano D. Baclofeno intratecal para el tratamiento de la espasticidad. Reporte de caso con revisión temática. Revista colombiana de anestesiología.2013; Vol.41 (3):229-235.

6. Dones I. Intrathecal baclofen for the treatment of spasticity. Acta Neurochirurgica Supplement. 2007 97(1): 185-188.

7. Vidal J. Tratamiento de la espasticidad mediante infusión intratecal de baclofeno. Instituto universitario de Neurorehabilitación Guttmann-UAB. Desarrollo de herramientas para evaluar el resultado de las tecnologías aplicadas al proceso rehabilitador. AATRM. 2006, Capítulo 11. Páginas: $85-88$.

8. Acevedo $\mathrm{J}$ Varón $\mathrm{L}$ Berbeo $\mathrm{M}$ et al. Avances fisiopatológicos para el entendimiento de la lesión medular traumática. Revisión bibliográfica. Revista Colombiana de Ortopedia y Traumatología. Diciembre 2008. Vol 22 (4): 272-281.

9. Vivancos F Pascual S Nardi J et al.Guía del tratamiento integral de la espasticidad. Revista Neurología. 2007, vol. 45 (6): 365-375.

10. Mertens P Parise M García L et al. Long-term clinical, electrophysiological and urodynamic effects of chronic intratecal baclofen infusión for treatment of spinal spasticity. Acta Neurochirurgica Supplement. 1995, 64: 17-25.

11. Koulousakis A Kuchta J. Intrathecal antispastic drug application with implantable pumps: results of a 10 year follow-up study. Acta Neurochirurgica Supplement. 2007, 97(1): 181184.

\section{CONFLICTO DE INTERÉS Y/O AGRADECIMIENTOS}

Los autores declaran que no existió ningún conflicto de interés en el presente reporte. 